\title{
TRANSIENTS FROM INITIAL CONDITIONS BASED ON LAGRANGIAN PERTURBATION THEORY IN N-BODY SIMULATIONS III: THE CASE OF GADGET-2 CODE
}

\author{
TAKAYUKI TATEKAWA \\ Department of Social Design Engineering, National Institute of Technology, Kochi College, \\ 200-1 Monobe-Otsu, Nankoku, Kochi, 783-8508, JAPAN \\ esearch Institute for Science and Engineering, Waseda University, 3-4-1 Okubo, Shinjuku, \\ Tokyo 169-8555, JAPAN \\ tatekawa@akane.waseda.jp \\ Received Day Month Year \\ Revised Day Month Year
}

\begin{abstract}
In modern cosmology, the precision of the theoretical prediction is increasingly required. In cosmological $N$-body simulations, the effect of higher-order Lagrangian perturbation on the initial conditions appears in terms of statistical quantities of matter density field. We have considered the effect of third-order Lagrangian perturbation (3LPT) on the initial conditions, which can be applied to Gadget-2 code. Then, as statistical quantities, non-Gaussianity of matter density field has been compared between cases of different order perturbations for the initial conditions. Then, we demonstrate the validity of the initial conditions with second-order Lagrangian perturbation (2LPT).
\end{abstract}

Keywords: Cosmology, large-scale structure, N-body simulations

02.60.Cb, 02.70.-c, 04.25.-g, 98.65.Dx

\section{Introduction}

Based on recent observations, refinement of the cosmological scenario is under progress ${ }^{1 / 5}$ For example, galaxy surveys not only present large-scale structures but also evolution of such structures in the Universe ${ }^{3 / 5}$ As the evolution of large-scale structures is clarified, various dark energy models,, 6 which explain the acceleration of the cosmic expansion, would be restricted.

As one of the useful methods to restrict cosmological models such as dark energy scenario, cosmological $N$-body simulations have been applied, $\stackrel{8]}{[13}$ which describe the evolution of nonlinear structures such as cluster of galaxies. Because cosmological $N$-body simulations include the cosmic expansion, evolution of the nonlinear structures would be affected by the dark energy models. By the comparison between observations and the predictions by the cosmological $N$-body simulations, we can verify the validity of dark energy models.

For precise verification of cosmological models such as dark energy model, precise simulations are required. We focus on the initial condition for cosmological 
$N$-body simulations, where Lagrangian linear perturbation, i.e., Zel'dovich approximation has been used for a long time. However, although Zel'dovich approximation describes the evolution of quasi-nonlinear density field, because it is described by linear perturbation, initial conditions fail to take into account higher-order growing modes.14]15 Recently, the effect of second-order Lagrangian perturbation (2LPT) on the initial condition for cosmological simulation has been studied, ${ }^{[16}$ which is manifested in the nonlinear structure at low-z era.

We investigated the effect of third-order Lagrangian perturbation (3LPT) on the initial condition for cosmological simulation. $\frac{17 \mid 18}{18}$ In the previous studies, we used $P^{3} M$ code for cosmological simulations 11 Although the execution speed of the simulation code is fast, $P^{3} M$ code can be applied for structure formation of cold dark matter only.

Gadget-2 $2^{13}$ is a well-known code for cosmological $N$-body/SPH simulation and can consider not only cold dark matter but also baryonic matter. The code can be executed on parallel computers with distributed memory. Therefore, huge simulations can be implemented with this code ${ }^{19}$ Hence, we have developed 3LPT initial condition code applicable to Gadget-2, which would be quite useful for various analyses considering several situations.

The effect of 3LPT on the initial condition is analysed in terms of statistical quantities for matter density field. Even if the initial condition is given by Gaussian distribution, the matter density field shows non-Gaussian distribution during nonlinear evolution. If the initial condition is set at $z=49$, the difference of the non-Gaussianity between the cases of 2LPT and 3LPT appears about $0.5 \%$. When we choose initial time at $z=99$, that difference almost disappear. From these results, we evaluate the effect of $3 \mathrm{LPT}$ for initial conditions and clarify the validity of initial conditions with 2LPT.

This paper is organized as follows. In Sec. 2, we present Lagrangian perturbations valid up to the third-order. Then, we discuss the methods and results of the numerical simulations in Sec. 3, In this section, we also introduce statistical quantities for matter distribution. Finally, Sec. 4 presents the conclusions.

\section{Lagrangian perturbations}

\section{1. basic equations}

In this section, we briefly introduce Lagrangian perturbation. When the scale of an object is smaller than that of the cosmological horizon, the description of motion of matter by Newtonian dynamics is valid. The cosmological expansion is affected by the scale factor $a$ in basic equations (continuous equation, Euler's equation, and Poisson's equation). The solution $a$ is derived by Friedmann's equations or alternative equations. We consider dust fluid, which can ignore the pressure of matter. In 
the comoving coordinates, the basic equations are described as follows $\underline{20} \underline{24}$

$$
\begin{aligned}
\frac{\partial \delta}{\partial t}+\frac{1}{a} \nabla_{x} \cdot\{\boldsymbol{v}(1+\delta)\} & =0, \\
\frac{\partial \boldsymbol{v}}{\partial t}+\frac{1}{a}\left(\boldsymbol{v} \cdot \nabla_{x}\right) \boldsymbol{v}+\frac{\dot{a}}{a} \boldsymbol{v} & =\frac{1}{a} \tilde{\boldsymbol{g}}, \\
\nabla_{x} \cdot \tilde{\boldsymbol{g}} & =-4 \pi G \bar{\rho} a \delta,
\end{aligned}
$$

where $\bar{\rho}$ represents background matter density. The density fluctuation $\delta$ is defined as

$$
\delta \equiv \frac{\rho-\bar{\rho}}{\bar{\rho}}
$$

$\boldsymbol{v}$ denotes peculiar velocity.

In Eulerian perturbation theory, the density fluctuation $\delta$ is regarded as a perturbation. On the other hand, in Lagrangian perturbation theory, displacement from a homogeneous distribution is considered as a perturbation. 24,27

$$
\boldsymbol{x}=\boldsymbol{q}+\boldsymbol{s}(\boldsymbol{q}, t)
$$

where $\boldsymbol{x}$ and $\boldsymbol{q}$ represent comoving Eulerian coordinates and Lagrangian coordinates, respectively. $s$ denotes the displacement vector, which is regarded as a perturbation quantity. By the Lagrangian perturbation (5), we can solve continuous equation (1) exactly.

$$
\delta=1-J^{-1}, J \equiv \operatorname{det}\left(\frac{\partial x_{i}}{\partial q_{j}}\right),
$$

$J$ refers to the Jacobian of the coordinate transformation from Eulerian $\boldsymbol{x}$ to Lagrangian $\boldsymbol{q}$. Therefore, when we derive the solution of Lagrangian displacement $s$, we can determine the evolution of the density fluctuation.

The peculiar velocity is given as

$$
\boldsymbol{v}=a \dot{\boldsymbol{s}} .
$$

We introduce Lagrangian time derivative

$$
\frac{\mathrm{d}}{\mathrm{d} t} \equiv \frac{\partial}{\partial t}+\frac{1}{a} \boldsymbol{v} \cdot \nabla_{x}
$$

Taking the divergence and rotation of Euler's equation (2), we obtain evolution equations for the Lagrangian displacement.

$$
\begin{aligned}
\nabla_{x} \cdot\left(\ddot{s}+2 \frac{\dot{a}}{a} \dot{s}\right) & =-4 \pi G \bar{\rho}\left(J^{-1}-1\right), \\
\nabla_{x} \times\left(\ddot{s}+2 \frac{\dot{a}}{a} \dot{s}\right) & =\mathbf{0} .
\end{aligned}
$$

Here, superscript dot $\dot{\boldsymbol{s}}$ refers to Lagrangian time derivative (8).

$$
\dot{s}=\frac{\mathrm{d} \boldsymbol{s}}{\mathrm{d} t} .
$$


To solve the Lagrangian perturbative equations, we decompose the Lagrangian perturbation into its longitudinal and transverse mode.

$$
\begin{gathered}
s_{i}=\psi_{, i}+\zeta_{i}, \\
\zeta_{i, i}=0 .
\end{gathered}
$$

where subscript ${ }_{i}$ denotes the Lagrangian spatial derivative.

We convert the spatial derivative from Eulerian coordinates to Lagrangian coordinates in equations (9) and (10).

$$
\begin{aligned}
\frac{\partial}{\partial x_{i}} & =\frac{\partial}{\partial q_{i}}-s_{j, i} \frac{\partial}{\partial x_{j}} \\
& =\frac{\partial}{\partial q_{i}}-s_{j, i} \frac{\partial}{\partial q_{j}}+s_{j, i} s_{k, j} \frac{\partial}{\partial x_{k}} \\
& =\frac{\partial}{\partial q_{i}}-s_{j, i} \frac{\partial}{\partial q_{j}}+s_{j, i} s_{k, j} \frac{\partial}{\partial q_{k}}+\cdots .
\end{aligned}
$$

where comma indicates Lagrangian spatial derivative.

$$
s_{j, i}=\frac{\partial s_{j}}{\partial q_{i}} .
$$

\subsection{Lagrangian perturbative equations}

In this subsection, we derive Lagrangian perturbative equations. The Lagrangian perturbation can be divided into temporal and spatial parts.

$$
\begin{aligned}
\psi & =g^{(1)} \psi^{(1)}+g^{(2)} \psi^{(2)}+g^{(3)} \psi^{(3)}+\cdots, \\
\zeta_{i} & =g^{(1 T)} \zeta_{i}^{(1)}+g^{(2 T)} \zeta_{i}^{(2)}+g^{(3 T)} \zeta^{(3)}+\cdots,
\end{aligned}
$$

where superscript ${ }^{(n)}$ denotes $n$-th order perturbation.

For the first-order perturbation, i.e., Zel'dovich approximation, 25 the differential equation for the temporal part is given as follows:

$$
\ddot{g}^{(1)}+2 \frac{\dot{a}}{a} \dot{g}^{(1)}-4 \pi G \bar{\rho} g^{(1)}=0 .
$$

When we consider only the growing mode of the temporal parts and set the temporal parts at the initial condition by $g^{(1)}\left(t_{\text {ini }}\right)=1$, the Lagrangian displacement is described by the density fluctuation.

$$
\psi_{, i i}^{(1)}(\boldsymbol{q})=-\delta(\boldsymbol{q}) .
$$

In other words, the first-order perturbation would be derived by the initial density fluctuation.

If the primordial vorticity does not exist, the vorticity never appears during evolution. Even if the primordial vorticity exists, the transverse mode in the firstorder perturbation does not have a growing solution. Therefore, hereafter we ignore the transverse mode in the first-order perturbation. 
The second-order perturbation is also divided into spatial and temporal parts. $\frac{28}{30}$ The equations are described as follows:

$$
\begin{aligned}
\psi_{, i i}^{(2)} & =\frac{1}{2}\left\{\psi_{, i i}^{(1)} \psi_{, j j}^{(1)}-\psi_{, i j}^{(1)} \psi_{, i j}^{(1)}\right\}, \\
\ddot{g}^{(2)}+2 \frac{\dot{a}}{a} \dot{g}^{(2)}-4 \pi G \bar{\rho} g^{(2)} & =-4 \pi G \bar{\rho}\left\{g^{(1)}\right\}^{2} .
\end{aligned}
$$

The third-order perturbation is derived from triplet term of the first-order perturbation and cross-section of the first- and the second-order perturbation 31 33

$$
\begin{aligned}
& \psi_{, i i}^{(3 a)}=\operatorname{det}\left(\psi_{, i j}^{(1)}\right) \\
&=\frac{1}{6} \psi_{, i i}^{(1)} \psi_{, j j}^{(1)} \psi_{, k k}^{(1)}-\frac{1}{2} \psi_{, i i}^{(1)} \psi_{, j k}^{(1)} \psi_{, j k}^{(1)}+\frac{1}{3} \psi_{, i j}^{(1)} \psi_{, j k}^{(1)} \psi_{, k i}^{(1)}, \\
& \psi_{, i i}^{(3 b)}=\frac{1}{2}\left\{\psi_{, i i}^{(1)} \psi_{, j j}^{(2)}-\psi_{, i j}^{(1)} \psi_{, i j}^{(2)}\right\}, \\
& \ddot{g}^{(3 a)}+2 \frac{\dot{a}}{a} \dot{g}^{(3 a)}-4 \pi G \bar{\rho} g^{(3 a)}=-8 \pi G \bar{\rho}\left(g^{(1)}\right)^{3}, \\
& \ddot{g}^{(3 b)}+2 \frac{\dot{a}}{a} \dot{g}^{(3 b)}-4 \pi G \bar{\rho} g^{(3 b)}=-8 \pi G \bar{\rho} g^{(1)}\left\{g^{(2)}-\left(g^{(1)}\right)^{2}\right\},
\end{aligned}
$$

Even if we do not consider transverse mode in the first-order perturbation, the transverse mode in the third-order appears 34

$$
\begin{aligned}
\ddot{g}^{(3 T)+}+2 \frac{\dot{a}}{a} \dot{g}^{(3 T)} & =4 \pi G \rho_{b}\left(g^{(1)}\right)^{3}, \\
-\nabla^{2} \zeta_{i}^{(3)} & =\left(\psi_{, i l}^{(1)} \psi_{, k l}^{(2)}-\psi_{, k l}^{(1)} \psi_{, i l}^{(2)}\right)_{, k} .
\end{aligned}
$$

Because of Kelvin's circulation theorem, the transverse mode in the third-order perturbation does not imply vorticity. In this paper, the effect of the transverse mode in the third-order perturbation is also analyzed.

In $\Lambda \mathrm{CDM}$ model, the early stage in structure formation is a matter dominant era. Because the effect of cosmological constant seems negligible, the cosmic expansion would be approximated by the solution of Einstein-de Sitter Universe model.

$$
a(t) \propto t^{2 / 3} .
$$

Under this assumption, the perturbative solutions become as follows:

$$
\begin{aligned}
g^{(1)}(t) & =t^{2 / 3}, \\
g^{(2)}(t) & =-\frac{3}{7} t^{4 / 3}, \\
g^{(3 a)}(t) & =\frac{10}{21} t^{2}, \\
g^{(3 b)}(t) & =-\frac{1}{3} t^{2},
\end{aligned}
$$


Bouchet et al $l^{[32}$ derived approximation formula of temporal parts for $\Lambda \mathrm{CDM}$ model. They introduced the logarithmic derivative of the growth factors

$$
f_{n}=\frac{a}{g^{(n)}} \frac{\mathrm{d} g^{(n)}}{\mathrm{d} a} .
$$

When the Universe is in the matter dominant era $\left(\Omega_{m} \simeq 1\right)$, the formula becomes

$$
\begin{gathered}
f_{1} \simeq \Omega_{m}^{6 / 11}, f_{2} \simeq 2 \Omega_{m}^{153 / 286}, \\
f_{3 a} \simeq 3 \Omega_{m}^{146 / 275}, f_{3 b} \simeq 3 \Omega_{m}^{9481 / 17875} .
\end{gathered}
$$

For the case of $0.1 \leq \Omega_{m} \leq 1$, the formula becomes

$$
\begin{gathered}
f_{1} \simeq \Omega_{m}^{5 / 9}, f_{2} \simeq 2 \Omega_{m}^{6 / 11}, \\
f_{3 a} \simeq 3 \Omega_{m}^{13 / 24}, f_{3 b} \simeq 3 \Omega_{m}^{13 / 24} .
\end{gathered}
$$

2LPT_IC code ${ }^{16}$ was implemented with the above formula.

\section{Cosmological Simulations}

\subsection{Setup of initial conditions}

For precise cosmological simulations, we set up precise initial conditions. For execution of Gadget-2, we developed a convert code of the initial conditions from ZA to 3LPT. The convert code is described in Sec 2.2. In 3LPT, analysis is performed separately for the presence or absence of the transverse mode. Hereafter, those without the transverse mode are described as "3LPT L", and those with the transverse mode are described as " $3 \mathrm{LPT} \mathrm{L}+\mathrm{T}$ ".

In this study, we set the initial condition at the redshift $z_{\text {ini }}=49$. Because the effect of the cosmological constant is negligible, the temporal components in the convert code are given by solutions of the Einstein-de Sitter model.

We set the cosmological parameters as shown in Table 1. The parameters of simulations are shown in Table 2. These parameters are sample values in the Gadget2 code, which are slightly different from the recent observation. 35

Table 1. Cosmological pa-
rameters in the simula-
tions.

Gadget-2 can be executed on many cores by OpenMPI. The simulation code was executed on Linux PC (CentOS 7.7, Core i9 7960X, RAM 64GB), using which the simulation can be executed for approximately 5 hours per one sample. 
Table 2. Parameters in the cosmological simulation code.

\begin{tabular}{lc}
\hline Initial time $z_{i n}$ & 49 \\
Box size $L$ & $100 h^{-1}[\mathrm{Mpc}]$ \\
Number of particles $N$ & $256^{3}$ \\
$h$ & 0.7 \\
Softening Length & $0.25 h^{-1}[\mathrm{Mpc}]$ \\
\hline
\end{tabular}

Our code was converted from the initial condition generated by ZA to that with 2LPT and 3LPT. Because our code cannot be executed parallelly, we cannot apply the code for huge simulations. For the case of $N=256^{3}$, the code requires about 2 GB memory. Especially in the calculation of the transverse mode in the third-order perturbation, the code occupies a large amount of memory.

In this simulation, we generated 10 initial conditions for each case. For the analysis of time evolution, we selected 11 time slices $(z=10,9, \cdots, 1,0)$. and compared the density distributions. The density field was smoothed over the scale $R$ using the cloud-in-a-cell (CIC) algorithm. The smoothing scale was set as $1 h^{-1}, 2 h^{-1}$ [Mpc].

\subsection{Non-Gaussianity}

For a detailed analysis, we apply the non-Gaussianity of the density fluctuation. Even if the primordial density fluctuation is generated by Gaussian distribution, The non-Gaussianity of the density fluctuation would appear through nonlinear evolution. For the analysis of the non-Gaussianity, we introduced higher-order statistical quantities:

$$
\begin{aligned}
\text { skewness : } \gamma & =\frac{\left\langle\delta^{3}\right\rangle}{\sigma^{4}}, \\
\text { kurtosis }: \eta & =\frac{\left\langle\delta^{4}\right\rangle-3 \sigma^{4}}{\sigma^{6}},
\end{aligned}
$$

where $\sigma$ means dispersion of the density fluctuation.

$$
\sigma=\sqrt{\left\langle\delta^{2}\right\rangle} .
$$

In the weakly nonlinear stage, these statistical quantities were derived by secondorder perturbation theory $20[26]$

\subsection{Effect of higher-order perturbations}

We analyze the effect of cosmological simulation when $3 \mathrm{LPT}$ is included in the initial conditions. In 3LPT, the effect of the transverse mode is extremely small, so the effect of the transverse mode is not discussed in this subsection. The effect of the transverse mode is analyzed in Section 3.6 .

First, we set the smoothing scale as about $1 h^{-1}[\mathrm{Mpc}]$. The distribution function of the density fluctuation is shown in Fig. 1. It is well-known that the distribution function of the density fluctuation approaches to log-normal form during the 


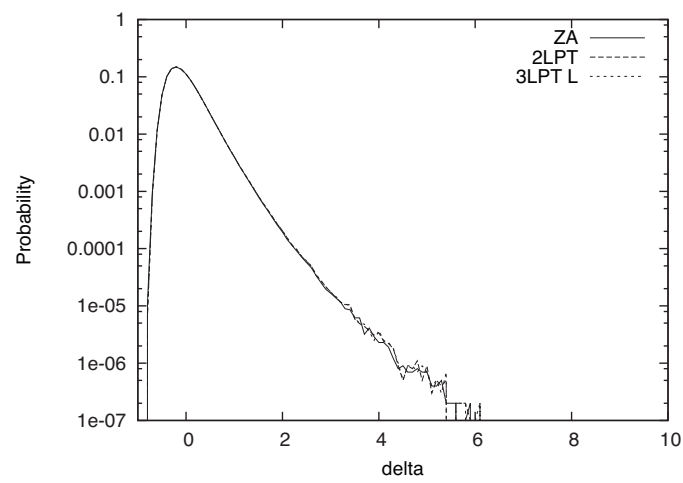

(a)

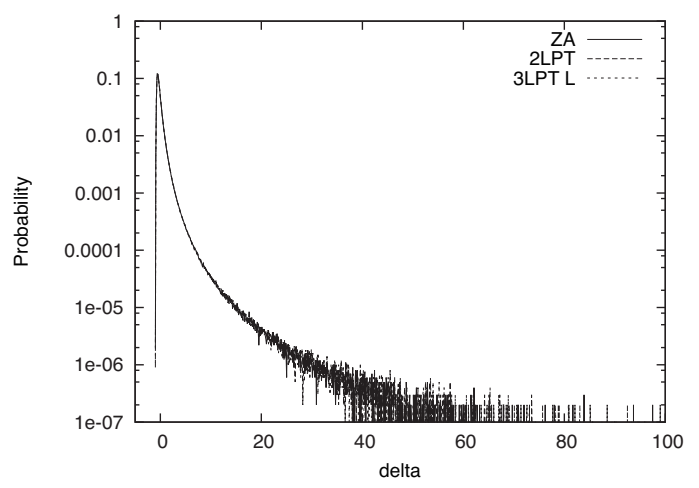

(c)

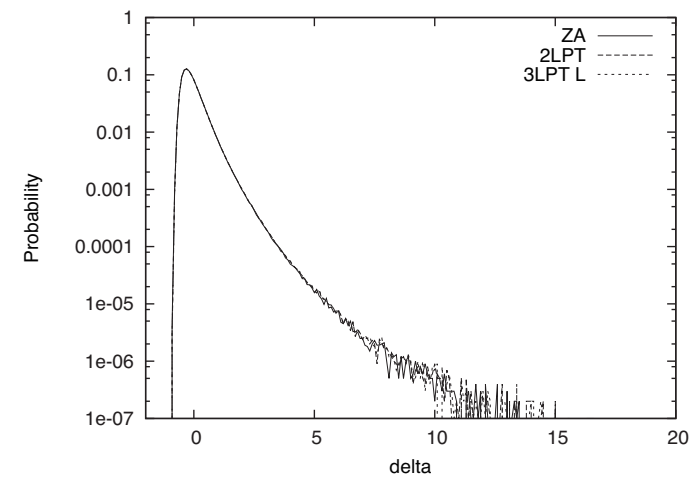

(b)

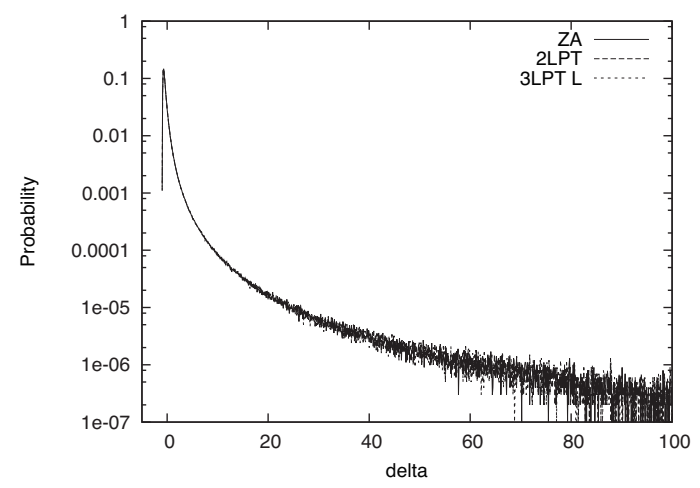

(d)

Fig. 1. Distribution function of the density fluctuation from $N$-body simulation $\left(R \simeq 1 h^{-1} \mathrm{Mpc}\right)$ with different initial conditions. (a) $z=5$, (b) $z=3$, (c) $z=1$, (d) $z=0$. The distribution function approaches the log-normal form during the evolution.

evolution $[36+39$ At $z=5$, the effect of higher-order perturbation appeared at the high-density region $\delta>10$. During the evolution, the high-density region grows rapidly. At $z=0$, the distribution functions resemble each other.

In our previous study, we showed the difference of the non-Gaussianity of the density fluctuation between the initial conditions given by ZA, 2LPT, and 3LPT. The difference between the cases of $2 \mathrm{LPT}$ and $3 \mathrm{LPT}$ is about several percent.

First, we show the evolution of the density dispersion with error bars. The evolution of the density dispersion is shown in Fig. 2. At $z=1$, the difference of the density dispersion between the case of ZA and 3LPT becomes about $2.5 \%$. Furthermore, the difference of the density dispersion between the case of $2 \mathrm{LPT}$ and $3 \mathrm{LPT}$ is about $0.2 \%$. We will notice the effect of transverse mode in 3LPT later.

Then, we show the evolution of the non-Gaussianity in Figs. 3 and 4 . Because variations among samples are very large, in the subsequent analysis, the error bars were omitted. By comparison between the case of ZA and higher-order perturba- 


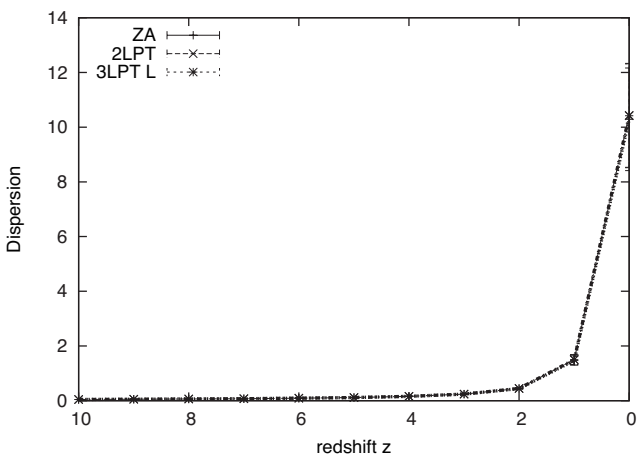

(a)

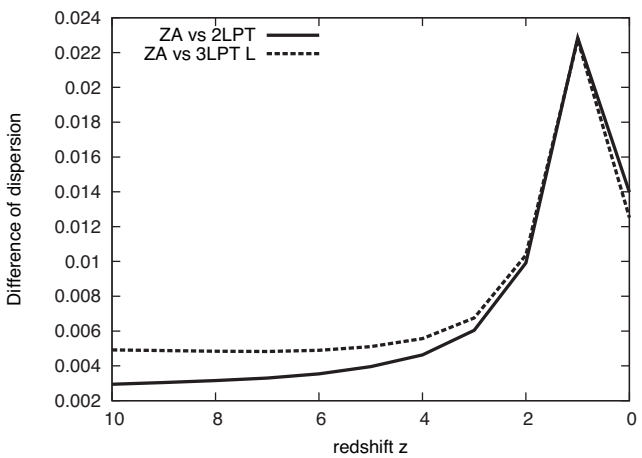

(b)

Fig. 2. Dispersion of the density fluctuation from $N$-body simulation $\left(R \simeq 1 h^{-1} \mathrm{Mpc}\right.$ ) with different initial conditions. (a) Comparison of the dispersion between the initial conditions. (b) The relative difference of the dispersion between $\mathrm{ZA}$ and other cases.

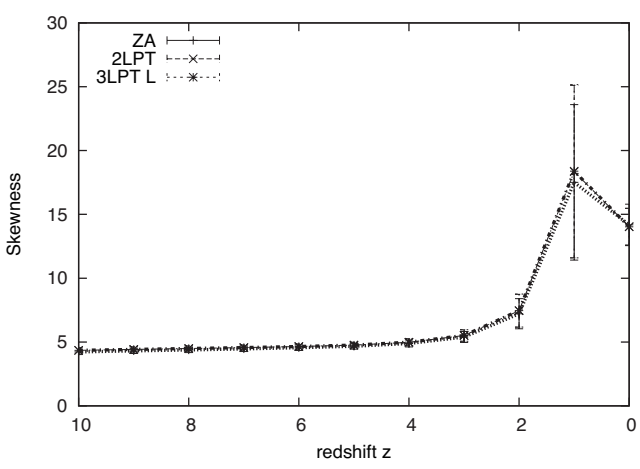

(a)

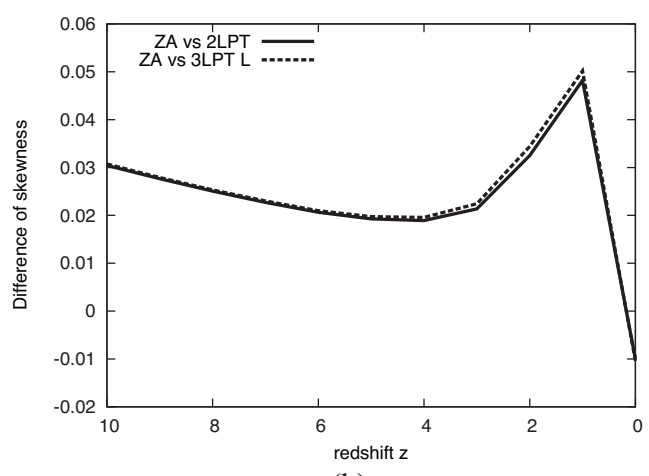

(b)

Fig. 3. Skewness of the density fluctuation from the $N$-body simulation $\left(R \simeq 1 h^{-1} \mathrm{Mpc}\right)$ with different initial conditions. (a) Comparison of the skewness between the initial conditions. (b) The relative difference in the skewness between $\mathrm{ZA}$ and other cases.

tions, the difference in the non-Gaussianity is about $5 \%$. When $z=0$, the difference in the non-Gaussianity between the cases of $2 \mathrm{LPT}$ and $3 \mathrm{LPT}$ becomes very small. The difference of skewness and kurtosis between the case of 2LPT and $3 \mathrm{LPT}$ is about $0.2 \%$ and $0.5 \%$, respectively. In this analysis, higher-order perturbations in the initial conditions affects the difference in the non-Gaussianity between models in high-z era $(z \simeq 10)$.

We change the smoothing scale to $R \simeq 2 h^{-1}$ [Mpc]. Even if the smoothing scale is changed, the tendency of the distribution function for the density fluctuation is similar to the case of $R \simeq 1 h^{-1}$ [Mpc]. The distribution function is shown in Fig. 5 .

Time evolution of dispersion of the density fluctuation is shown in Fig. 6] The non-Gaussianity of the distribution of the density fluctuation is shown in Figs. 7 and 8 . The difference of skewness and kurtosis between the case of 2LPT and 3LPT 


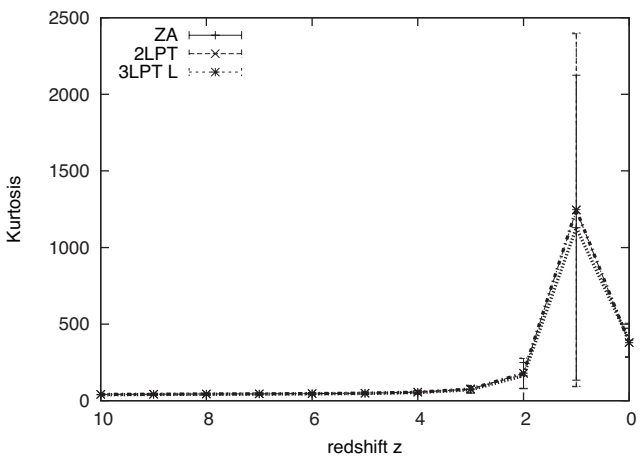

(a)

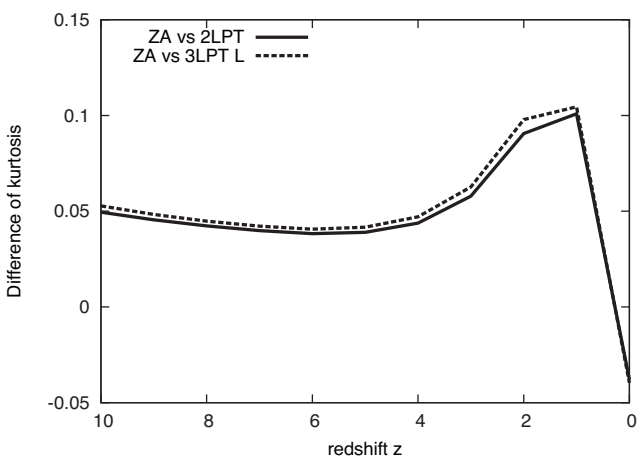

(b)

Fig. 4. Kurtosis of the density fluctuation from the $N$-body simulation $\left(R \simeq 1 h^{-1}[\mathrm{Mpc}]\right)$ with different initial conditions. (a) Comparison of the kurtosis between the initial conditions. (b) The relative difference in the kurtosis between $\mathrm{ZA}$ and other cases.

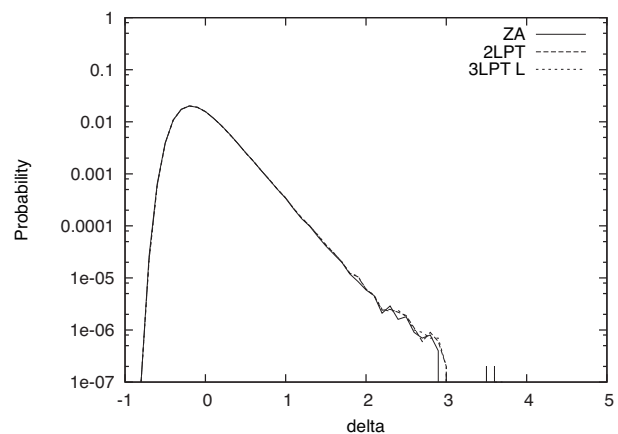

(a)

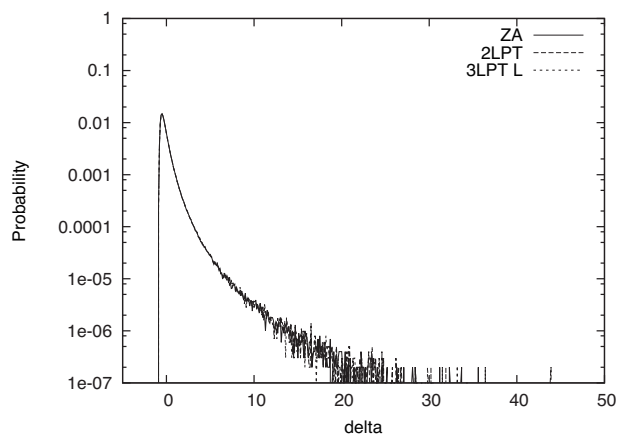

(c)

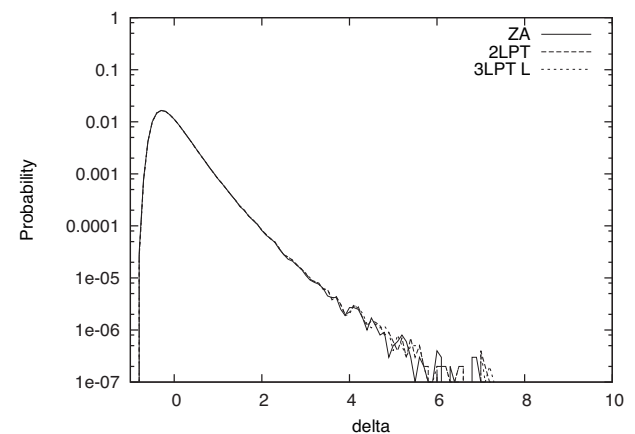

(b)

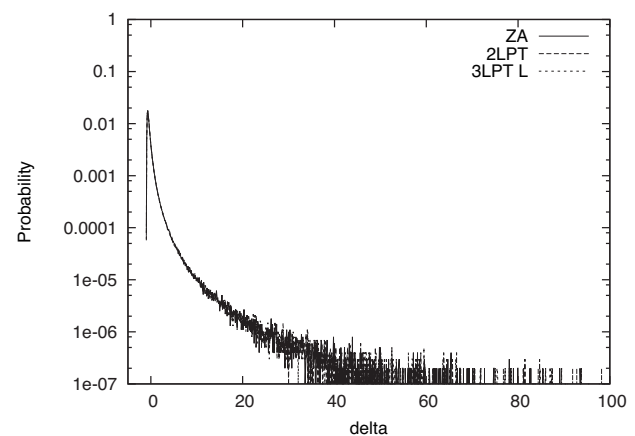

(d)

Fig. 5. Distribution function of the density fluctuation from the $N$-body simulation $\left(R \simeq 2 h^{-1}\right.$ Mpc) with different initial conditions. (a) $z=5$, (b) $z=3$, (c) $z=1$, (d) $z=0$. The distribution function approaches the log-normal form during the evolution. 


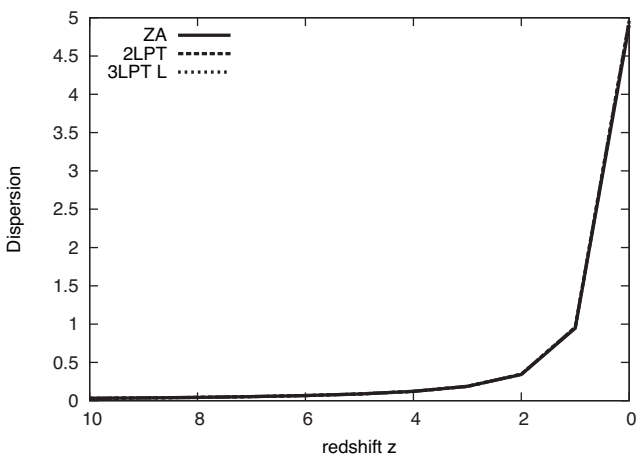

(a)

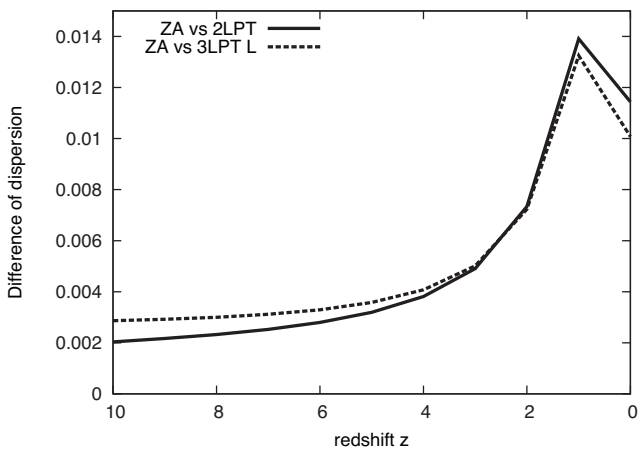

(b)

Fig. 6. Dispersion of the density fluctuation from the $N$-body simulation $\left(R \simeq 2 h^{-1} \mathrm{Mpc}\right)$ with different initial conditions. (a) Comparison of the dispersion between the initial conditions. (b) The relative difference in the dispersion between ZA and other cases.

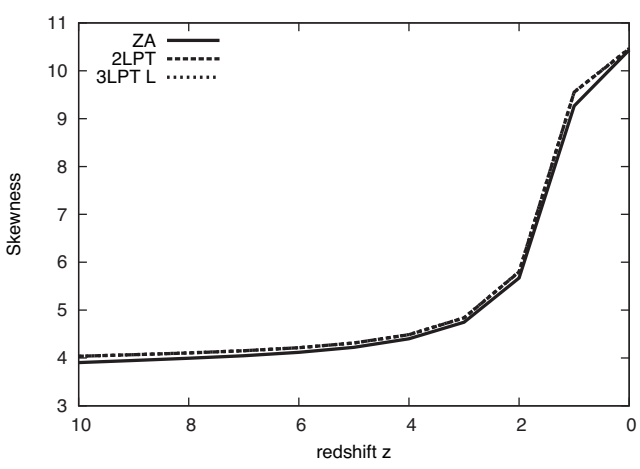

(a)

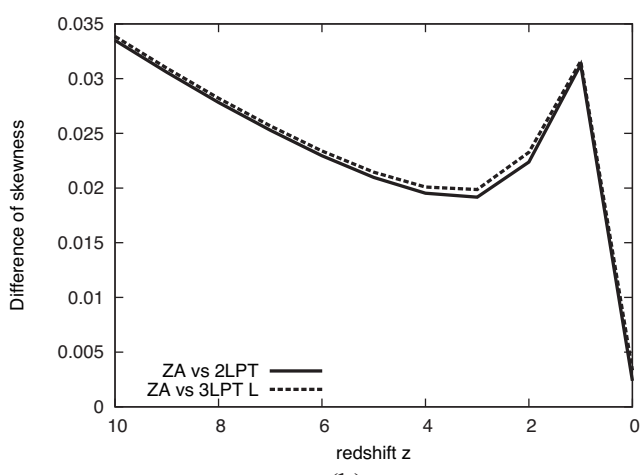

(b)

Fig. 7. Skewness of the density fluctuation from the $N$-body simulation $\left(R \simeq 2 h^{-1} \mathrm{Mpc}\right)$ with different initial conditions. (a) Comparison of the skewness between the initial conditions. (b) The relative difference in the skewness between $\mathrm{ZA}$ and other cases.

is about $0.1 \%$ and $0.3 \%$, respectively.

We noticed a distribution of peculiar velocity. Here, we compute the absolute value of the peculiar velocity for each particle. When clusters are formed, the particles in clusters slow down. Therefore, the peculiar velocity does not increase monotonically. The probability of the peculiar velocity for each time is shown in Fig. 9 . The effect of higher-order perturbation in the initial conditions appears in fast particles. For a more detailed analysis, we compare the probability of the peculiar velocity between the case of ZA and other cases. The difference in the probability of the peculiar velocity is shown in Fig. 10. The effect of higher-order perturbation appears in fast particles. When we consider higher-order perturbation for the initial conditions of the $N$-body simulation, the peculiar velocity increases. At $z=5$, the number of fast particles (faster than $500[\mathrm{~km} / \mathrm{s}]$ ) in the case of $2 \mathrm{LPT}$ is more than 


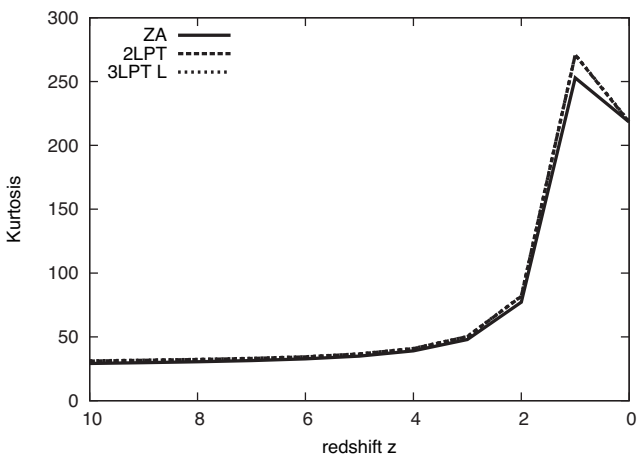

(a)

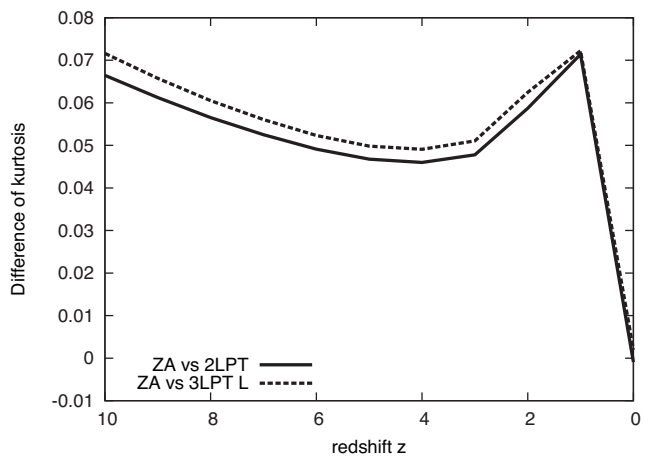

(b)

Fig. 8. Kurtosis of the density fluctuation from the $N$-body simulation $\left(R \simeq 2 h^{-1}\right.$ [Mpc]) with different initial conditions. (a) Comparison of the kurtosis between initial conditions. (b) The relative difference in the kurtosis between $\mathrm{ZA}$ and other cases.

that in the case of ZA. Similarly, the number of fast particles in the case of 3LPT is more than that in the case of $2 \mathrm{LPT}$. Although the particles form clusters at low- $z$ era $(z=3,1,0)$, the tendency continues afterwards. It was found that higher-order perturbations increased the number of fast particles, but the number of fast particles was small compared to the whole. Even if the density distribution is considered in the redshift space instead of the real space, the effect of higher-order perturbations on the deformation by the peculiar velocity would be small.

\subsection{Dependence on initial time}

The effect of higher-order perturbations increases during time evolution. If the initial conditions for $N$-body simulation set on early stage, will the effect of higher-order perturbation weaken? To verify this conjecture, we change the initial time for $\mathrm{N}$ body simulation to $z=99$. In this simulation, we generated 10 initial conditions for each case (ZA, 2LPT, and 3LPT L). The way of allocating pseudo-random numbers when creating the initial conditions is the same as that for cases of $z=49$. Fig. 111 shows the evolution of the density dispersion. Compared to the case of $z=49$, the growth of dispersion is slightly slower for the case of $z=99$.

We show the evolution of the non-Gaussianity in Figs. 12 and 13 By comparison between the case of ZA and higher-order perturbations, the difference in the skewness and the kurtosis is less than $5 \%$ and $12 \%$, respectively. Compared to the case of $z_{\text {ini }}=49$, the difference between the models is smaller when $z_{\text {ini }}=99$, but the non-Gaussian difference between ZA and 2LPT remains several percents. By comparison between the case of $2 \mathrm{LPT}$ and $3 \mathrm{LPT}$, the difference in the kurtosis is less than $0.5 \%$. In this analysis, the effect of higher-order perturbations in the initial conditions disappears in the non-Gaussianity at high-z era $(z \simeq 10)$. On the other hand, the effect remains in the non-Gaussianity at low-z era $(z<2)$. 


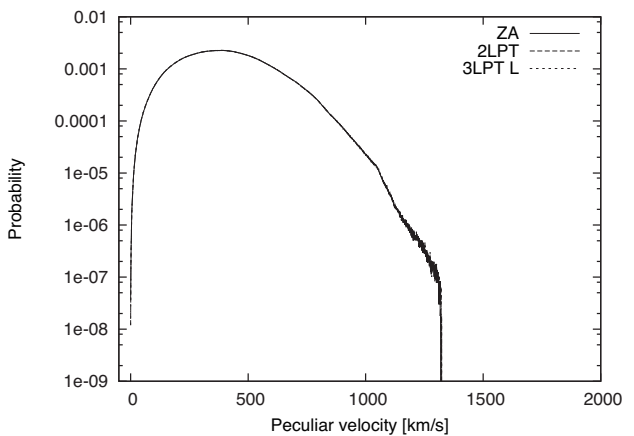

(a)

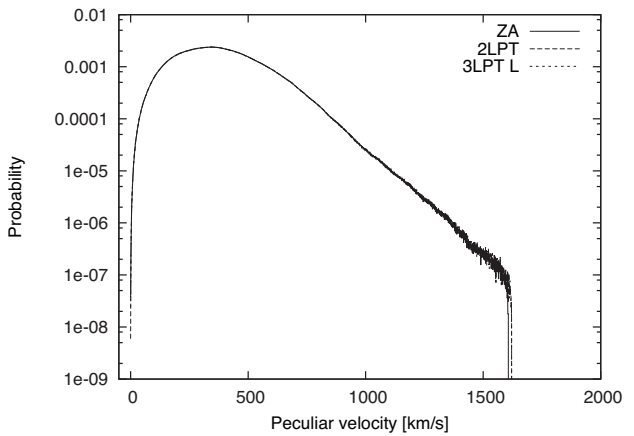

(c)

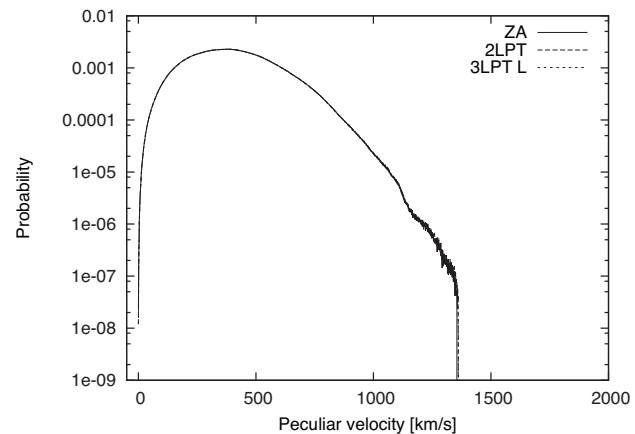

(b)

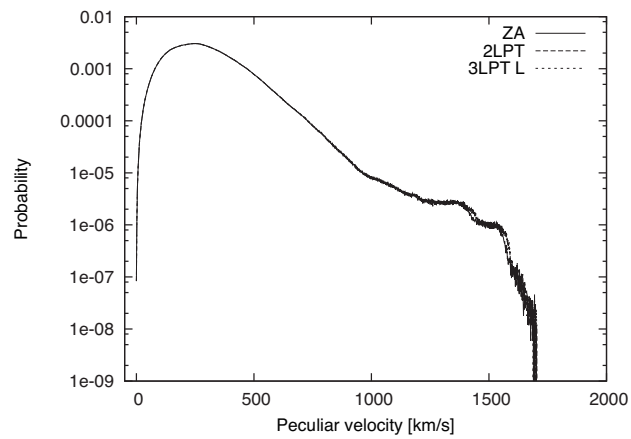

(d)

Fig. 9. The distribution function of the peculiar velocity from the $N$-body simulation with different initial conditions. (a) $z=5$, (b) $z=3$, (c) $z=1$, (d) $z=0$. At high-z era, the effect of higher-order perturbation in the initial conditions appears.

\subsection{Dependence on number of particles}

For cosmological $N$-body simulations, the characteristics of the formed structure may be affected by the difference in the number of particles. In this paper, in order to avoid the effect of the number of particles, we perform simulations with a large number of particles. The number of particles is increased to $N=512^{3}$. Since this simulation takes a long time (about 60 hours), only 7 samples were executed in this paper. The other parameters are the same as those listed in Table 1 and 2 In this simulation, we generated 7 initial conditions for each case (ZA, 2LPT, and 3LPT L). Fig. 14 shows the evolution of the density dispersion. Compared to the case of $N=256^{3}$, Compared to the case of $N=256^{3}$, the tendency of evolution is similar in the case of $N=512^{3}$. The difference in the density dispersion between models is similar for cases of $N=512^{3}$ and $N=256^{3}$. By comparison between the case of $\mathrm{ZA}$ and higher-order perturbations, the difference in the density dispersion is about $10 \%$. The difference of the density dispersion between the case of $2 \mathrm{LPT}$ and $3 \mathrm{LPT}$ is about $1 \%$. The variation in the density dispersion appears because the number of samples is small. 


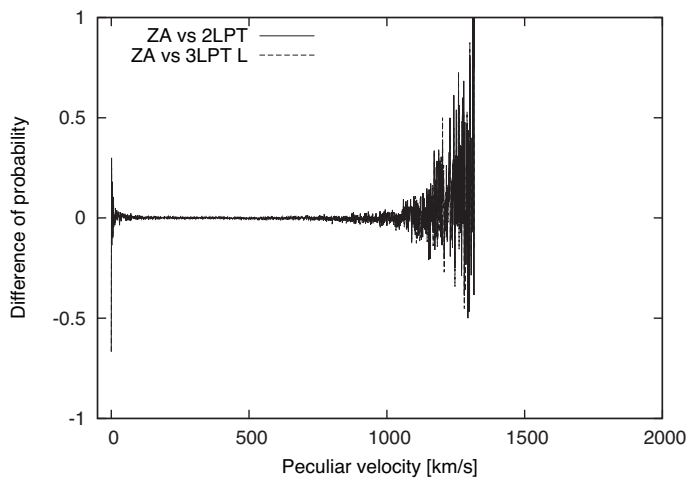

(a)

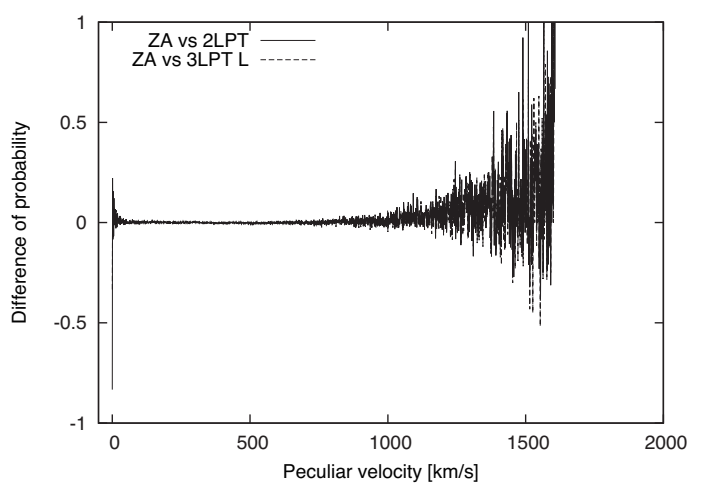

(c)

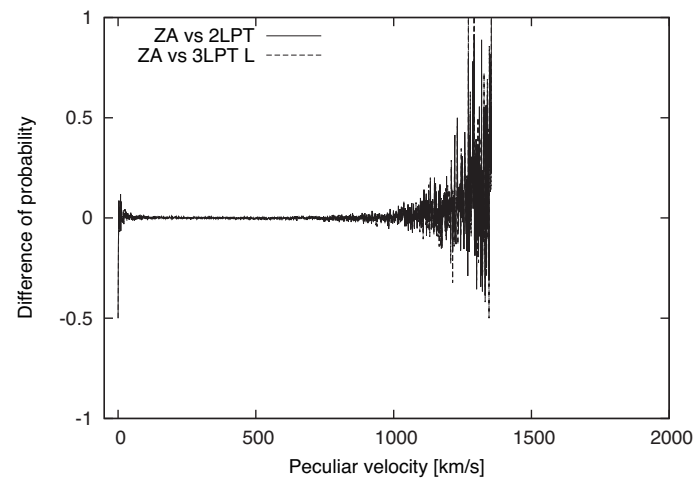

(b)

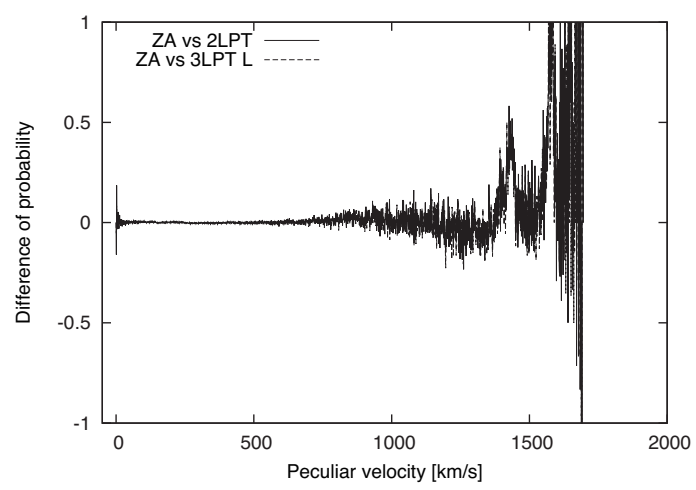

(d)

Fig. 10. The relative difference in the distribution function of the peculiar velocity between the case of ZA and other cases. (a) $z=5$, (b) $z=3$, (c) $z=1$, (d) $z=0$.

We show the evolution of the non-Gaussianity in Figs. 15 and 16. In the case of $N=512^{3}$, the same evolution tendency as in the case of $N=256^{3}$ can be seen. Regarding the evolution of the non-Gaussianity, the differences between the models are similar for cases of $N=512^{3}$ and $N=256^{3}$. By comparison between the case of $\mathrm{ZA}$ and higher-order perturbations, the difference in the non-Gaussianity is about $10 \%$. When $z=0$, the difference in the non-Gaussianity between the cases of 2LPT and 3LPT becomes very small. The difference of skewness and kurtosis between the case of $2 \mathrm{LPT}$ and $3 \mathrm{LPT}$ is about $0.4 \%$ and $1 \%$, respectively. The variation in non-Gaussianity appears because the number of samples is small.

From the above results, it was shown that the effect of higher-order perturbations in the initial condition is almost independent of the number of particles.

\subsection{Effect of the transverse mode in $3 L P T$}

We examine how the presence or absence of the transverse mode in 3LPT affects nonlinear structure. We compare the non-Gaussianity of the density fluctuation with 


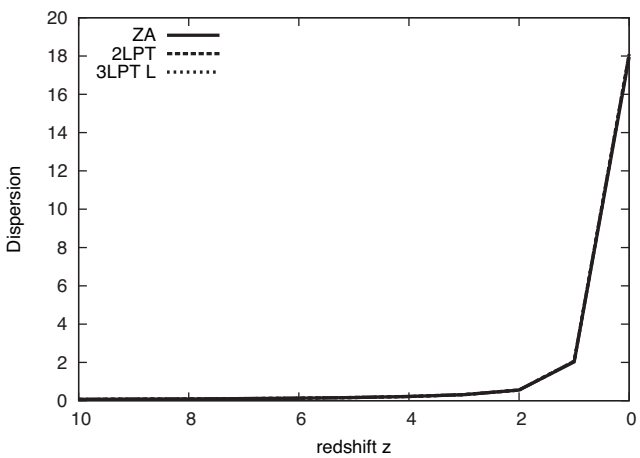

(a)

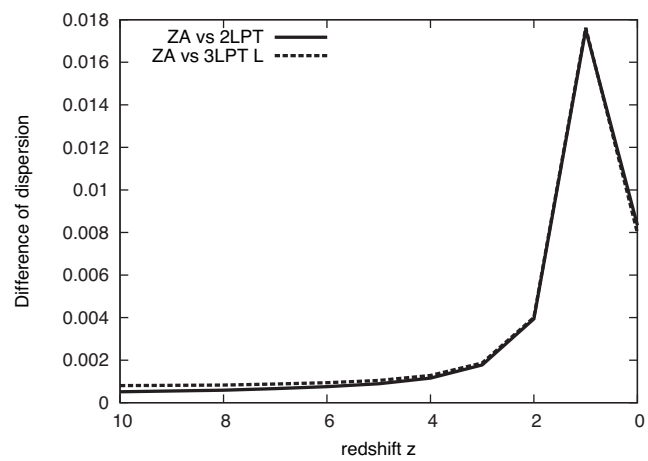

(b)

Fig. 11. Dispersion of the density fluctuation from $N$-body simulation $\left(R \simeq 1 h^{-2} \mathrm{Mpc}, z_{\text {ini }}=99\right)$ with different initial conditions. (a) Comparison of the dispersion between the initial conditions. (b) The relative difference of the dispersion between ZA and other cases.

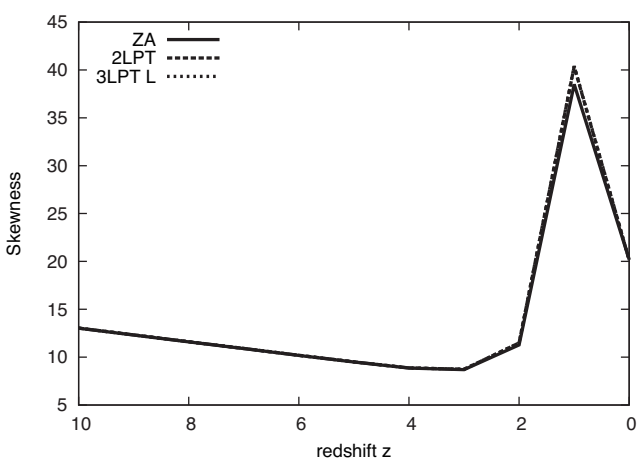

(a)

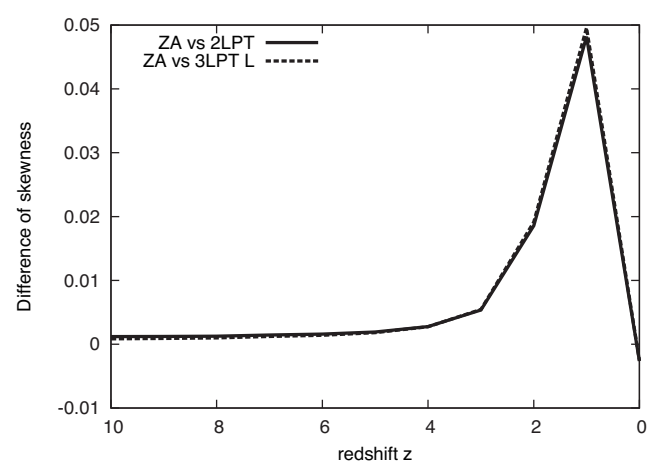

(b)

Fig. 12. Skewness of the density fluctuation from the $N$-body simulation $\left(R \simeq 1 h^{-1} \mathrm{Mpc}, z_{\text {ini }}=\right.$ 99) with different initial conditions. (a) Comparison of the skewness between the initial conditions. (b) The relative difference in the skewness between ZA and other cases.

and without the transverse mode in 3LPT ("3LPT L" vs "3LPT L+T"). Figure 17 shows how much the non-Gaussianity of the density fluctuation that includes the transverse mode deviates from the one that does not include the transverse mode. In any case, the difference between 2LPT and 3LPT is much smaller and negligible.

The reason can be explained using Eq. (6). Eq. (6) can be rewritten as follows.

$$
\begin{aligned}
\delta= & -\left(s_{i, i}+\frac{1}{2}\left(s_{i, i} s_{j, j}-s_{i, j} s_{i, j}\right)+\operatorname{det}\left(s_{i, j}\right)\right) \\
& \cdot\left(1+s_{i, i}+\frac{1}{2}\left(s_{i, i} s_{j, j}-s_{i, j} s_{i, j}\right)+\operatorname{det}\left(s_{i, j}\right)\right)^{-1} .
\end{aligned}
$$




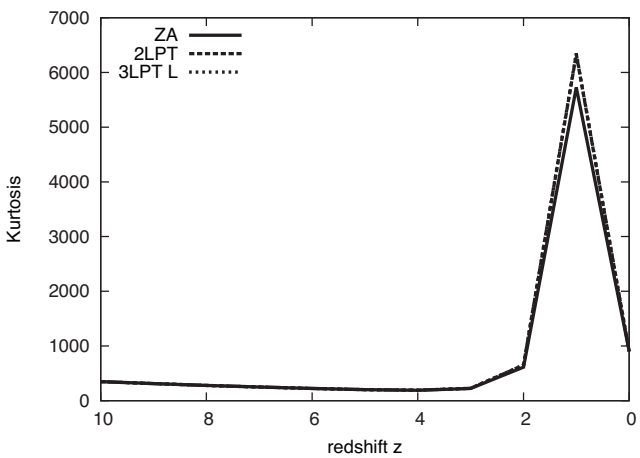

(a)

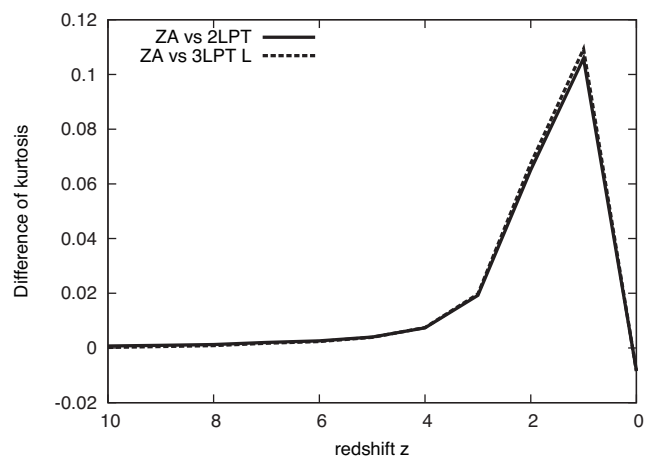

(b)

Fig. 13. Kurtosis of the density fluctuation from the $N$-body simulation $\left(R \simeq 1 h^{-1}\right.$ [Mpc], $\left.z_{\text {ini }}=99\right)$ with different initial conditions. (a) Comparison of the kurtosis between the initial conditions. (b) The relative difference in the kurtosis between ZA and other cases.

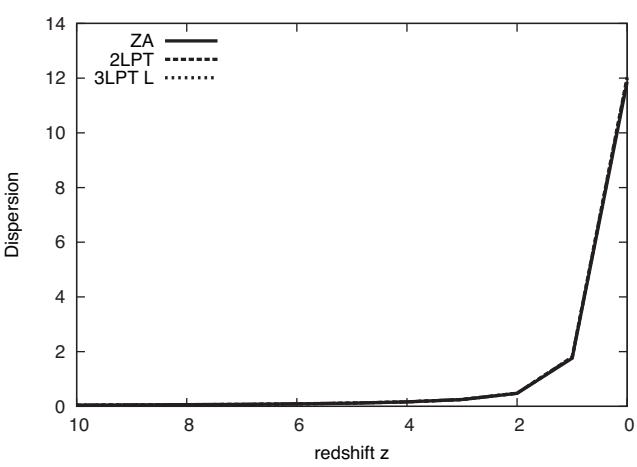

(a)

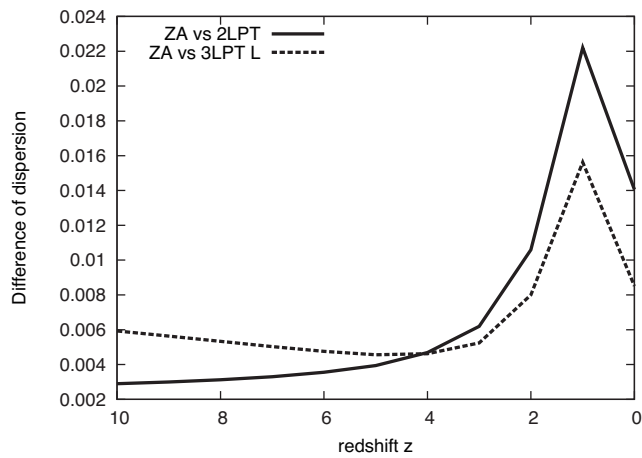

(b)

Fig. 14. Dispersion of the density fluctuation from N-body simulation $\left(R \simeq 1 h^{-2} \mathrm{Mpc}, N=512^{3}\right)$ with different initial conditions. (a) Comparison of the dispersion between the initial conditions. (b) The relative difference of the dispersion between ZA and other cases.

This equation is expanded to the order of perturbation.

$$
\begin{aligned}
\delta= & -\psi_{, i i}^{(1)} \\
& +\left[-\psi_{, i i}^{(2)}+\frac{1}{2}\left(\psi_{, i i}^{(1)} \psi_{, j j}^{(1)}+\psi_{, i j}^{(1)} \psi_{, i j}^{(1)}\right)\right] \\
& +\left[-\psi_{, i i}^{(3)}+\left(\psi_{, i i}^{(1)} \psi_{, j j}^{(2)}+\psi_{, i j}^{(1)} \psi_{, i j}^{(2)}\right)-\psi_{, i i}^{(1)} \psi_{, j k}^{(1)} \psi_{, j k}^{(1)}-\operatorname{det}\left(\psi_{, i j}^{(1)}\right)\right] \\
& +\left[\psi_{, i j}^{(1)}\left(\psi_{, i j}^{(3)}+\zeta_{i, j}^{(3)}\right)+F_{4}\left(\psi_{, i j}^{(1)}, \psi_{, i j}^{(2)}, \psi_{, i j}^{(3)}\right)\right]+O\left(\varepsilon^{5}\right),
\end{aligned}
$$

where $F_{4}$ means fourth-order perturbative quantity which composed of first-, second-, and third-order perturbation in the longitudinal mode. $O\left(\varepsilon^{5}\right)$ means fifthor more higher-order perturbative quantities. The transverse mode appears in fourth-order term. Therefore, at least the transverse mode has only a fourth-order 


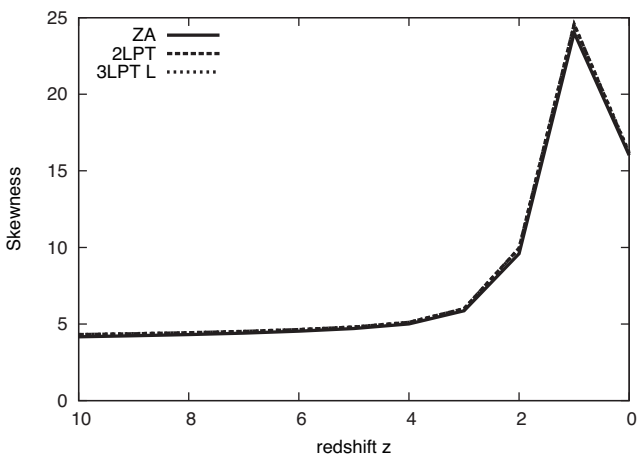

(a)

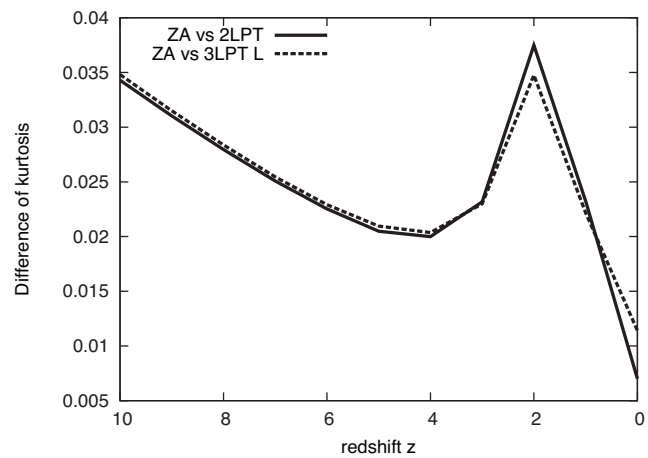

(b)

Fig. 15. Skewness of the density fluctuation from the N-body simulation $\left(R \simeq 1 h^{-1} \mathrm{Mpc}\right.$, $N=512^{3}$ ) with different initial conditions. (a) Comparison of the skewness between the initial conditions. (b) The relative difference in the skewness between ZA and other cases.

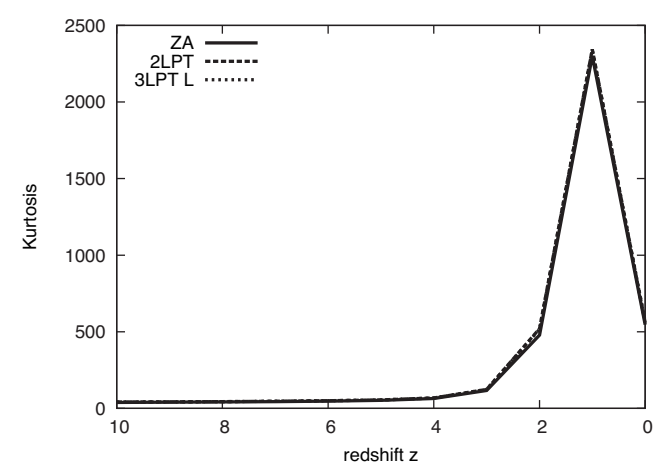

(a)

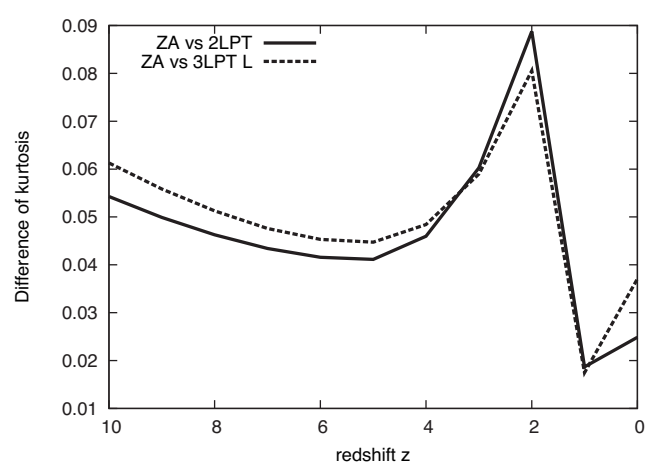

(b)

Fig. 16. Kurtosis of the density fluctuation from the N-body simulation $\left(R \simeq 1 h^{-1}[\mathrm{Mpc}], N=\right.$ $512^{3}$ ) with different initial conditions. (a) Comparison of the kurtosis between the initial conditions.

(b) The relative difference in the kurtosis between ZA and other cases.

or higher-order effect on density fluctuations.

The effect of the transverse mode clearly appears on the velocity distribution. Figure 18 shows how the difference between the presence and absence of the transverse mode in the initial conditions appears in the velocity distribution. The probability distribution of fast velocities varies greatly between them.

\subsection{Comparison with past research}

The validity of this study is compared with past studies. Therefore, we compare our results with those using 2LPT_IC code. In this paper, we examine the nonGaussianity of the density distribution between 2LPT_IC code and our code. Figure 19 shows how much it deviates from ZA. For each of these quantities, the case of 2LPT_IC code shows a slightly larger deviation than the case of our code. This 


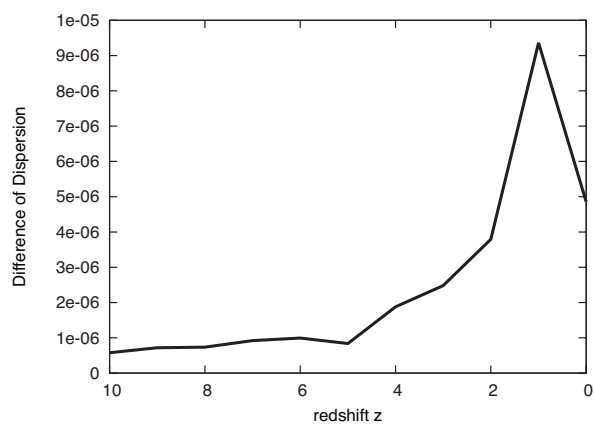

(a)

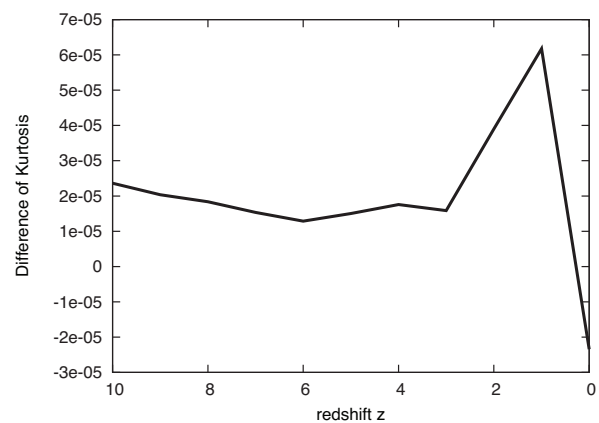

(c)

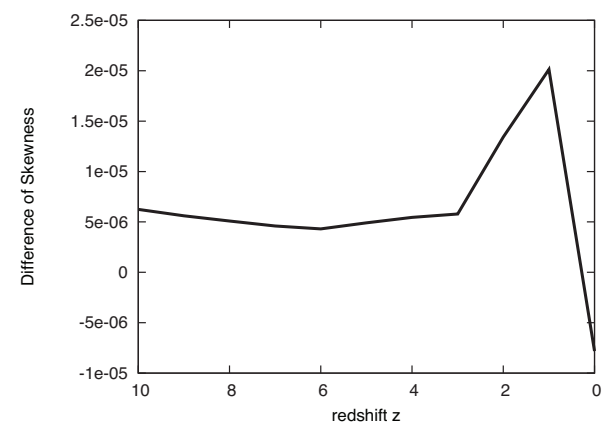

(b)

Fig. 17. The relative difference of non-Gaussianity with and without the transverse mode $(R \simeq$ $1 h^{-1}[\mathrm{Mpc}]$ ). This figure shows how much it shifts when the transverse mode is included. (a) Dispersion, (b) skewness, (c) kurtosis.

result shows a larger deviation than when the order of the perturbation was raised to the third-order in our code.

The difference between the two codes is thought to depend on the method used to solve Poisson equation. In 2LPT_IC code, the derivative of the perturbation is calculated in Fourier space to solve Poisson equation. On the other hand, in our code, the derivative of the perturbation is calculated in real space to solve Poisson equation. It is thought that the difference between the two methods caused the difference. The perturbation growth rate is not corrected by the density parameter in our code, but it seems that this effect is small at $z_{\text {ini }}=49$. On the contrary, the growth of higher-order perturbations in our code is larger than these in 2LPT_IC code.

\section{Summary}

We analysed the effect of higher-order perturbation for the initial conditions of cosmological $N$-body simulations. Based on our previous studies, we developed an initial condition converter for Gadget-2 code. 


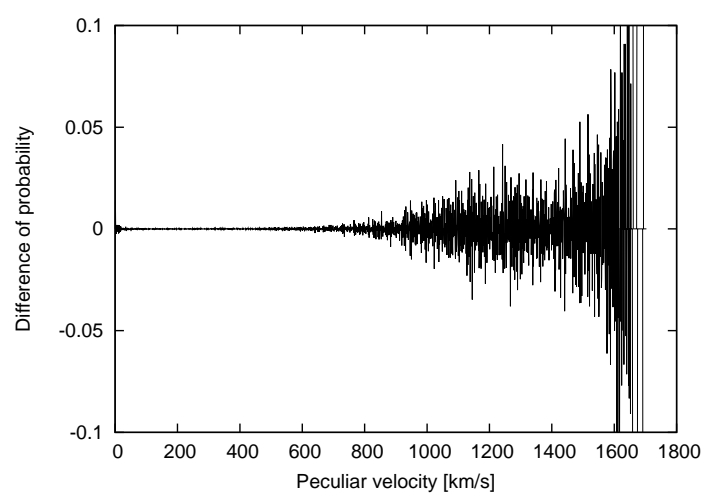

Fig. 18. The relative difference in the distribution function of the peculiar velocity between the presence and absence of the transverse mode $(z=0)$. The probability distribution of fast velocities varies greatly between them.

For density fluctuation, the effect of higher-order perturbation appeared in strongly nonlinear region. Although the primordial density fluctuation was generated by the Gaussian distribution, because of nonlinear evolution, the nonGaussianity of the distribution of the density fluctuation appeared at low- $z$ era. Further, we compared the statistical quantities for the non-Gaussianity. Although it varied significantly depending on the samples, the effect of 3LPT on the initial condition was evident. Even if the time of the initial condition is set early, although the effect of 3LPT would disappear, the effect of 2LPT affects the evolution of the density distribution later. It was also clarified that the effect of higher-order perturbations hardly depends on the number of particles in the simulation.

With regard to the peculiar velocity, the higher-order perturbation affected fast particles, and during clustering, the effect gradually disappeared. Considering the density distribution in redshift space, the effect of higher-order perturbation would appear in the shape of structures such as finger-of-god 40,41

For the 2LPT initial conditions, the results obtained using our code were slightly different from that obtained by the 2LPT_IC code. In our code, the spatial differential was calculated by the difference in the Lagrangian space. On the other hand, in the 2LPT_IC code, the spatial differential was calculated in the Lagrangian Fourier space. Further, in our code, the time evolution from recombination era to the initial time $\left(z_{\text {ini }}=49\right)$ was given by the growing factor in the E-dS Universe mode. In the 2LPT_IC code, the time evolution was given by an approximated formula for the $\Lambda \mathrm{CDM}$ model. The difference in the initial set up spread in the nonlinear stage.

This study shows that it is appropriate to apply initial conditions including 2LPT when the accuracy of $3 \mathrm{LPT}$ is unnecessary. Although the results obtained 


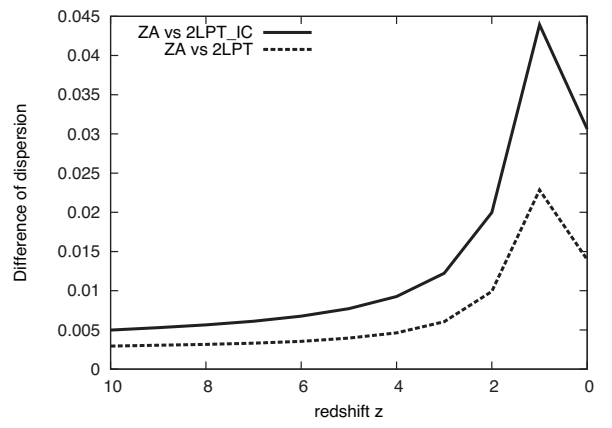

(a)

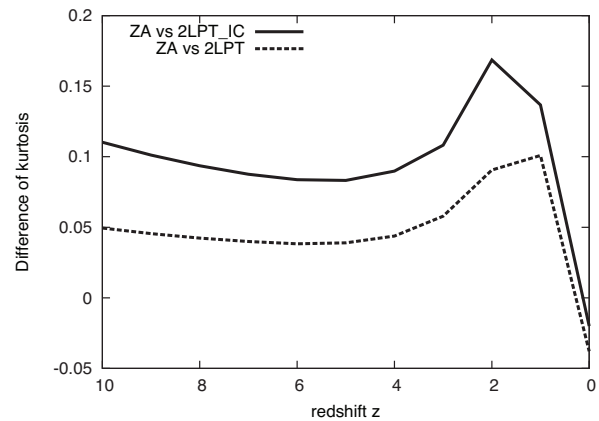

(c)

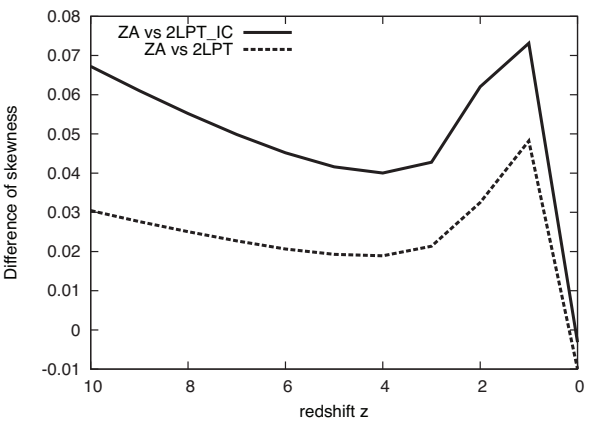

(b)

Fig. 19. The relative difference of the density distribution between 2LPT_IC code and our code $\left(R \simeq 1 h^{-1} \mathrm{Mpc}\right)$. This figure shows the difference between the case of ZA and each code. (a) Dispersion, (b) skewness, (c) kurtosis.

using both the codes varied slightly, we demonstrated that the effect of $3 \mathrm{LPT}$ in the initial condition appeared in the nonlinear stage. Therefore, for more precise prediction for a large-scale structure (with $0.1 \%$ accuracy), the effect of 3LPT on the initial condition should be considered for cosmological $N$-body simulations. This effect would appear in other statistical quantities as well.

\section{Acknowledgments}

We thank Shuntaro Mizuno and Toshihiro Nishimichi for useful comments.

\section{References}

\section{References}

1. M. Colless et al., Mon. Not. R. Astron. Soc. 328 (2001) 1039.

2. K. N. Abazajian et al., Astrophys. J. Supp. 182 (2009) 2.

3. LSST Science Collaborations, arXiv:0912.0201.

4. E. Sánchez E (forthe Des collaboration), J. Phys. Conf. Ser. 259 (2010) 012080.

5. R. Laureijs et al., arXiv:1110.3193. 
6. P. J. E. Peebles and B. Ratra, Rev. Mod. Phys., 75 (2003) 559.

7. E. J. Copeland, M. Sami and S. Tsujikawa, Int. J. Mod. Phys. D, 15 (2006) 1753.

8. K. Miyoshi and T. Kihara, Pub. Astron. Soc. Japan 27 (1975) 333.

9. A. Klypin and S. F. Shandarin, Mon. Not. R. Astron. Soc. 204 (1983) 891.

10. G. Efstathiou, M. Davis, C. S. Frenk and S. D. M. White, Astrophys. J. Suppl. 57 (1985) 241.

11. W. R. Hockney and W. Eastwood, Computer Simulation Using Particles (Taylor \& Francis Group, New York, 1988); E. Bertschinger and J. M. Gelb, Computers in Physics 5 (2) (1991) 164.

12. E. Bertschinger, Ann. Rev. Astron. Astrophys. 36 (1998) 599.

13. V. Springel, Mon. Not. R. Astron. Soc. 364 (2005) 1105.

14. R. Scoccimarro, Mon. Not. Roy. Astron. Soc. 299 (1998) 1097.

15. P. Valageas, Astron. Astrophys. 385 (2002) 761.

16. M. Crocce, S. Pueblas and R. Scoccimarro, Mon. Not. R. Astron. Soc. 373 (2006) 369.

17. T. Tatekawa and S. Mizuno, J. Cosmol. Astropart. Phys. 12 (2007) 014.

18. T. Tatekawa, J. Cosmol. Astropart. Phys. 04 (2014) 025.

19. V. Springel et al., Nature 435 (2005) 629.

20. P. J. E. Peebles, The Large-Scale Structure of the Universe (Princeton: Princeton University Press, 1980).

21. A. R. Liddle and D. H. Lyth, Cosmological Inflation and Large-Scale Structure (Cambridge: Cambridge University Press, 2000).

22. P. Coles and F. Lucchin, Cosmology, The Origin and Evolution of Cosmic Structure (Chichester: John Wiley, 2002).

23. S. Weinberg, Cosmology (Oxford University Press, Oxford, 2008).

24. V. Sahni and P. Coles, Phys. Rep. 262 (1995) 1.

25. Ya. B. Zel'dovich, Astron. Astrophys. 5 (1970) 84.

26. F. Bernardeau, S. Colombi, E. Gaztañaga and R. Scoccimarro, Phys. Rept. 367 (2002) 1 .

27. T. Tatekawa, Recent Res. Devel. Astrophys. 2 (2005) 1. arXiv:astro-ph/0412025.

28. F. R. Bouchet, R. Juszkiewicz, S. Colombi and R. Pellat, Astrophys. J. 394 (1992) L5.

29. T. Buchert and J. Ehlers, Mon. Not. R. Astron. Soc. 264 (1993) 375.

30. D. Munshi, V. Sahni and A. A. Starobinsky, Astrophys. J. 436 (1994) 517.

31. T. Buchert, Mon. Not. R. Astron. Soc. 267 (1994) 811.

32. F. R. Bouchet, S. Colombi, E. Hivon and R. Juszkiewicz, Astron. Astrophys. 296 (1995) 575.

33. P. Catelan, Mon. Not. R. Astron. Soc. 276 (1995) 115.

34. M. Sasaki and M. Kasai, Prog. Theor. Phys. 99 (1998) 585.

35. Planck colalboration, arXiv:1807.06209

36. A. Hamilton, Astrophys. J. 292 (1985) L35.

37. L. Kofman, E. Bertschinger, J. M. Gelb, A. Nusser and A. Dekel, Astrophys. J. 420 (1994) 44.

38. I. Kayo, A. Taruya and Y. Suto, Astrophys. J. 561 (2001) 22.

39. J. P. Ostriker, K. Nagamine, R. Cen and M. Fukugita, Astrophys. J. 597 (2003) 1.

40. J. C. Jackson, Mon. Not. R. Astron. Soc. 156 (1972) 1.

41. R. Scoccimarro, Phys. Rev. D 70 (2004) 083007. 